Check for updates

Cite this: RSC Adv., 2020, 10, 17101

\section{A high-throughput and untargeted lipidomics approach reveals new mechanistic insight and the effects of salvianolic acid B on the metabolic profiles in coronary heart disease rats using ultra- performance liquid chromatography with mass spectrometry $\dagger$}

\author{
Ying-peng Li,\$ Cong-ying Wang, \$ Hong-tao Shang, Rui-rui Hu, Hui Fu (DD * \\ and Xue-feng Xiao*
}

\begin{abstract}
High-throughput lipidomics provides the possibility for the development of new therapeutic drugs. Accordingly, herein, we reveal the protective role of salvianolic acid B (Sal B) in rats with coronary heart disease (CHD) and propose a new mechanism for its action through a high-throughput and nontargeted lipidomics strategy. A CHD animal model was induced by consecutive high-fat diet feeding with vitamin D3 injection. At the end of the 8th week, the serum sample was analyzed to explore the metabolic biomarker and pathway changes using untargeted lipidomics based on ultra-performance liquid chromatography with mass spectrometry (UPLC/MS). In addition, blood and heart tissue samples were collected and processed for the detection of biochemical indicators and liver histological observation. After salvianolic acid B treatment, the levels of LDH, CK, CK-MB, MYO, CTn1, TG, TC, LDL-C, and Apo(b) were significantly lower than that in the model group, while the levels of HDL-C and Apo(a1) were significantly higher than that in the model group. Furthermore, the histological features of fibrosis and steatosis were also evidently relieved in the model group. A total of twenty-six potential biomarkers were identified to express the lipid metabolic turbulence in the CHD animal models, of which twentytwo were regulated by salvianolic acid $B$ trending to the normal state, including TG(20:0/20:4/0-18:0), PC(20:4/18:1(9Z)), PC(18:3/20:2), PA(18:0/18:2), LysoPE(18:2/0:0), SM(d18:0/22:1), PE(22:6/0:0), LysoPE (20:4/0:0), sphinganine, Cer(d18:0/18:0), PS(14:0/14:1), PC (18:0/16:0), LysoPC(17:0), PE(22:2/20:1), $\mathrm{PC}(20: 3 / 20: 4), \mathrm{PE}(20: 4 / \mathrm{P}-16: 0), \mathrm{PS}(20: 3 / 18: 0)$, cholesterol sulfate, $\mathrm{TG}(15: 0 / 22: 6 / 18: 1)$, prostaglandin E2, arachidonic acid and sphingosine-1-phosphate. According to the metabolite enrichment and pathway analyses, the pharmacological activity of salvianolic acid B on CHD is mainly involved in three vital metabolic pathways including glycerophospholipid metabolism, sphingolipid metabolism and arachidonic acid metabolism. Thus, based on the lipidomics-guided biochemical analysis of the lipid biomarkers and pathways, Sal B protects against CHD with good therapeutic effect by regulating glycerophospholipid metabolism, sphingolipid metabolism and arachidonic acid metabolism, inhibiting oxidative stress damage and lipid peroxidation.
\end{abstract}

Received 3rd January 2020 Accepted 12th April 2020

DOI: $10.1039 / \mathrm{d} 0 \mathrm{ra00049c}$ rsc.li/rsc-advances
Currently, it is the cause of the highest morbidity and mortality in the world, resulting in 7.4 million deaths globally, and is predicted that there will be even more patients suffering from CHD in 2020. ${ }^{2,3}$ The principal clinical manifestations of CHD are myocardial infarction (MI), palpitation, and angina pectoris, which are associated with unhealthy lifestyle behaviors, age, gender, and some psychosocial risk factors. ${ }^{4,5}$ Some epidemiologic studies have indicated that the early intervention and treatment of CHD can hinder approximately $75 \%$ of cardiac events and slow disease progression and even death. ${ }^{6}$ The main clinical treatment methods are medication, such as nitrates,
Tianjin University of Traditional Chinese Medicine, Tianjin, 301617, China. E-mail: biomarker1973@163.com; fhmedical@126.com

$\dagger$ Electronic supplementary information (ESI) available. See DOI: 10.1039/d0ra00049c

\$ These authors contributed equally. 
antithrombotic drugs and $\beta$-blockers, percutaneous coronary angioplasty, and coronary artery bypass grafting. However, despite the decline in mortality from CHD in recent years, it is still a huge health and economic burden in society. ${ }^{7}$ Furthermore, the potential molecular mechanism of $\mathrm{CHD}$ remains unclear. Therefore, there is an urgent need for in-depth research and the development of novel therapeutic strategies for the treatment of CHD.

Natural products have become the focus in the global research community since the Chinese Scientist Youyou $\mathrm{Tu}$ discovered artemisinin, which is an anti-malaria wonder drug, and was awarded the 2015 Nobel Prize in Physiology or Medicine..$^{\mathbf{8} 9}$ As the representative component of phenolic acids derived from the dried root and rhizome of Salvia miltiorrhiza Bge (Labiatae), salvianolic acid B (Sal B) accounts for $3-5 \%$ of the total dry weight of this herb and exhibits a variety of remarkable and reliable pharmacological activities, such as inhibiting atherosclerosis, remitting myocardial ischemia/ reperfusion injury, and defending the nervous system. ${ }^{\mathbf{1 0 - 1 2}}$ It is an index ingredient for the content determination of Salvia miltiorrhiza Bunge. According to Chinese Pharmacopoeia 2015 editions, the pure product of Sal B is a white powder with poor thermal stability, which is easily soluble in water, methanol, and ethanol. Due to the presence of nine phenolic hydroxyls in the chemical structure of $\mathrm{Sal} \mathrm{B}$, it has the ability to provide $\mathrm{H}^{+}$to block lipid peroxidation in cells, and its antioxidant activity is superior to that of glutathione, vitamin E, and Ginkgo biloba extract. ${ }^{13}$ Several studies have indicated that Sal B is highly effective for the treatment of patients with cardiovascular disease, cerebrovascular disease, Alzheimer's disease, Parkinson's disease, and renal injury. ${ }^{\mathbf{1 4}}$ In the treatment of cardiovascular disease, it plays a significant role in promoting cardiac angiogenesis, protecting myocardial cells from apoptosis, inhibiting left ventricular remodeling and ischemia of myocardial injury, improving hemorheology, preventing cell migration and proliferation, and intimal hyperplasia. ${ }^{\mathbf{1 5}}$ However, the specific underlying mechanism for the therapeutic effect of Sal B on CHD is still poorly understood.

Some studies have shown that lipid metabolism devastation is inextricably related to the occurrence and development of various human diseases, such as apoptosis, signal transduction, disease infection, and immune function and fetal metabolic defects. ${ }^{\mathbf{1 6}}$ The capability to remove cholesterol becomes weaker with a decrease in the level of high-density lipoprotein cholesterol, triggering early-onset CHD, and triglyceride-rich lipoproteins also play a major role in causing atherosclerosis. ${ }^{\mathbf{1 7}}$ Lipids play a critical role biological membranes, such as maintaining the membrane structure, storing energy, messenger molecules and regulating effect. ${ }^{18}$ In 2003, the concept of lipidomics was proposed by researchers, which is an area of the metabolomics research field for probing the species and biological function of lipid molecules on a largescale and complete molecular level. ${ }^{19}$ Research on the identification and quantification of lipids involves fatty acids, glycerophospholipids, glycerolipids, sphingolipids, sterols, saccharolipids, prenol lipids and others in cells, tissues, and biological fluids. It methodically expresses the small changes in the composition and expression of lipids triggered by stimuli in vivo and vitro, which offers a novel opportunity for comprehending the functional activity of various lipids in disease. ${ }^{20,21}$ Furthermore, the use of powerful instruments such LC/MS and their combination with advanced informatics technologies has accelerated the characterization of a wide variety of lipids under metabolic conditions. In a relatively short analysis time, hundreds of lipids from total lipid extract have been directly identified and precisely quantified. ${ }^{22}$ Several studies have reported that the lipidomics-based approach is conducive to risk prediction and therapeutic monitoring in cardiovascular disease through the discovery of potential biomarkers and pathways. Apparently, it is an effective strategy for the in-depth study of chemical materials and understand the efficacy of natural products. ${ }^{23}$ Patients with CAD show high levels of ceramides, diglycerides, saturated, and unsaturated monoglycerides and a low content of unsaturated TG than patients without CAD in the epicardial adipose tissue (EAT) ${ }^{\mathbf{2 4 - 2 6}}$ Herein, a high-throughput and untargeted lipidomics method based on UPLC/MS was employed to investigate the lipid metabolic profile changes in rats with CHD caused by a high-fat diet with vitamin D3 injection after salvianolic acid B administration, which revealed the pharmacological activity of salvianolic acid $B$ and the mechanism for its protection against CHD, demonstrating the application value of lipidomics for the early detection, prediction, treatment and prognosis of CHD patients as a new platform for drug discovery.

\section{Materials and methods}

\subsection{Chemicals and reagents}

Vitamin D3 injection (specifications $1 \mathrm{~mL}: 7.5 \mathrm{mg}$, National Pharmaceutical Standard H31034121) was obtained from ATCC (Shanghai, China). The high-fat diet including 2\% cholesterol, $0.5 \%$ sodium cholate, $3 \%$ lard oil, $0.2 \%$ propylthiouracil, and 94.3\% basic diet was prepared in the laboratory for the study. Pentobarbital sodium and sodium chloride injection were obtained from Shanghai Chemical Reagent Purchasing and Supply Station, China. Formaldehyde solution was obtained from Shanghai General Pharmaceutical Co., Ltd, China. The salvianolic acid B standard was purchased from North China Pharmaceutical, China. The lactate dehydrogenase (LDH), creatine kinase (CK) and creatine kinase-MB (CK-MB) kits were obtained from Ningbo Medical System Biotechnology Co., Ltd (Ningbo, China). Myoglobin (MYO), troponin I (CTn1), triglyceride (TG) and total cholesterol (TC) were purchased from Steraloids, Inc. (Newport, RI). The low density lipoprotein cholesterin (LDL-c) and high density lipoprotein cholesterin (HDLc) kits were purchased from Cayman Chemical Company (Ann Arbor, MI). The apolipoprotein b (Apob) and apolipoprotein A1 (Apoa1) kits were obtained from Cayman Chemical (Ann Arbor, MI, USA). Chromatographic grade formic acid, acetonitrile, and methanol were obtained from Merck (KGaA Merck). Distilled water was obtained from Guangzhou Watsons Food \& Beverage Co., Ltd, China. Leucine enkephalin was purchased from Sigma-Aldrich (St Louis, MO, USA). Additional reagents and drugs met the experimental requirements. 


\subsection{Experimental animals}

A total of thirty-six male Sprague-Dawley rats (clean-grade, age: 6-8 weeks, body weight: $200 \pm 20 \mathrm{~g}$ ) were purchased from the Laboratory Animal Centre of Tianjin University of Traditional Chinese Medicine. All the experimental rats were housed under controlled conditions, where room temperature was maintained at $20 \pm 2{ }^{\circ} \mathrm{C}$ with $50 \pm 5 \%$ humidity and normal circadian rhythm. Animals were supplied with free access to food and water. All experimental procedures were approved by the Animal Care and Ethics Committee at Tianjin University of Traditional Chinese Medicine and performed in accordance with the declaration of Helsinki.

\subsection{CHD model preparation}

According to the preliminary experimental results, we adopted the following method to prepare the CHD model preparation. After the adaptive feed was administered for seven days, thirtysix rats were randomly divided into the control group, CHD model group and Sal B treatment group. The rat model of CHD was induced with vitamin D3 injection once every day $\left(1.5 \times 10^{6}\right.$ $\mathrm{U} \mathrm{kg}^{-1}$ ) and fed with a high-fat diet containing $2 \%$ cholesterol, $0.5 \%$ sodium cholate, $3 \%$ lard oil, $0.2 \%$ propylthiouracil, and $94.3 \%$ basic diet in all but the control group. The control group was given a normal diet. The total experimental period was eight weeks.

\subsection{Salvianolic acid B treatment}

From the first day of modeling, the rats in the Sal B treatment group were intragastrically administered a Sal B solution daily (concentration: $1.5 \mathrm{mg} \mathrm{mL}^{-1}$; $20 \mathrm{~mL}$ per kg body weight), and the control and model group received and equivalent dose of distilled water ( $20 \mathrm{~mL}$ per $\mathrm{kg}$ body weight) in the same way.

\subsection{Histopathological and biochemical index evaluation}

On the last day of the 8th week, all the animals were subjected to fasting overnight and then anesthetised with $30 \mathrm{mg} \mathrm{kg} \mathrm{kg}^{-1}$ pentobarbital intravenously $\left(2.0 \mathrm{~mL} \mathrm{~kg}^{-1}\right.$ body weight $)$ the next day. Approximately $5 \mathrm{~mL}$ blood sample was taken from the hepatic portal vein and transferred to a tube with $3.8 \%$ sodium citrate. The blood sample was then centrifuged for $15 \mathrm{~min}$ at $12000 \mathrm{rpm}$ and $4{ }^{\circ} \mathrm{C}$, and the obtained supernatant, named serum, was collected and stored at $-80{ }^{\circ} \mathrm{C}$ for the detection biochemical indicators related to CHD and lipidomics analysis. The clinical biochemistry kits including LDH, CK, CK-MB, MYO, CTn1, TG, TC, HDL-c, LDL-c, Apo(b) and Apo(a1) were used according to the manufacturer's instructions. The color and texture of the heart were observed macroscopically, and it was placed in a $10 \%$ neutral formalin solution for $24 \mathrm{~h}$ for hematoxylin-eosin (HE) staining.

\subsection{Lipidomics analysis}

2.6.1 Preparation of lipid extracts from serum samples. The serum samples were thawed on ice at $4{ }^{\circ} \mathrm{C}$ for $60 \mathrm{~min}$ before lipid extraction. The lipid internal standard was dissolved in methanol solution and then mixed with $50 \mu \mathrm{L}$ serum sample at a ratio of $8: 1$. The mixture was added $1000 \mu \mathrm{L}$ methyl tert-butyl ether and $400 \mu \mathrm{L}$ water. After vortexing for $30 \mathrm{~s}$ and centrifuging at $12000 \mathrm{rpm}$ for $10 \mathrm{~min}$ at $4{ }^{\circ} \mathrm{C}, 400 \mu \mathrm{L}$ supernatant was obtained and transferred to a centrifuge tube for lyophilization. The residue was added to $40 \mu \mathrm{L}$ dichloromethane-methanol (ratio of $2: 1$ ) and then $150 \mu \mathrm{L}$ acetonitrile-isopropanol-water (ratio of $65: 30: 5$ ) containing $5 \mathrm{mmol} \mathrm{L}^{-1}$ ammonium acetate to dissolve it. After centrifuging at $13000 \mathrm{rpm}$ for $15 \mathrm{~min}$ at $4{ }^{\circ} \mathrm{C}$, the supernatant was filtered using a $0.22 \mu \mathrm{m}$ filter membrane for UPLC/MS analysis. The quality control (QC) sample contained $20 \mu \mathrm{L}$ of solution from each of serum sample, which was injected three times at the beginning of the run in UPLC/MS operation and every seven samples were tested again to ensure the reliability of the data.

2.6.2 UPLC/MS analysis of lipids. The UPLC/MS system was based on an Accela UPLC (Thermo Fisher Scientific Corporation, Waltham, MA, USA) coupled with an LTQ-Orbitrap XL hybrid mass spectrometer (linear ion trap mass spectrometer, Orbitrap). A BEH C18 analytic column $(2.1 \times 100 \mathrm{~mm}, 1.7 \mu \mathrm{m}$, Waters, Milford, MA, USA) and a binary solvent system (solvent A: acetonitrile with $0.1 \%$ formic acid and solution B: water with $0.1 \%$ formic acid) were employed for the separation of lipids via reversed-phase chromatography. The gradient elution condition was programmed as follows: $0-2 \mathrm{~min}$, from $5 \%$ to $15 \%$ solution $\mathrm{A} ; 2-5 \mathrm{~min}$, from $15 \%$ to $48 \%$ solution $\mathrm{A} ; 5-7.5 \mathrm{~min}$, from $48 \%$ to $70 \%$ solution $\mathrm{A} ; 7.5-$ $10 \mathrm{~min}$, from $70 \%$ to $100 \%$ solution A; and then hold at $100 \%$ for $2 \mathrm{~min}$. The lipid extracts of the serum were analyzed at a flow rate of $0.4 \mathrm{~mL} \mathrm{~min}{ }^{-1}$, the column temperature was fixed at $40{ }^{\circ} \mathrm{C}$ and the injection volume was set as $5 \mu \mathrm{L}$. Meanwhile, the optimized parameters of the tandem mass spectrometer were set as follows: the capillary voltage was $3.5 \mathrm{kV}$ and the desolvation gas flow was $1500 \mathrm{~L} \mathrm{~h}^{-1}$ in positive mode and the capillary voltage was $3.0 \mathrm{kV}$ and the desolvation gas flow was $800 \mathrm{~L} \mathrm{~h}^{-1}$ in negative mode. The other main parameters were the same, including sampling cone voltage: $23 \mathrm{~V}$, source temperature: $120{ }^{\circ} \mathrm{C}$, desolvation temperature: $300{ }^{\circ} \mathrm{C}$, cone gas flow: $55 \mathrm{~L} \mathrm{~h}^{-1}$, nebulizer: $6.8 \mathrm{Bar}$, and ramp trap collision energy: $25-35 \mathrm{~V}$. The $\mathrm{m} / \mathrm{z}$ range was from 50 to 1500 . $200 \mathrm{pg} \mathrm{mL}{ }^{-1}$ leucine-enkephalin calibration solution in $[\mathrm{M}-\mathrm{H}]-$ $=554.2615$ and $[\mathrm{M}+\mathrm{H}]-=556.2771$ at $5 \mu \mathrm{L} \mathrm{m^{-1 }}$ was employed to retain the preciseness and reproducibility of the MS. The scan time and inter-scan time were $0.2 \mathrm{~s}$ and $0.02 \mathrm{~s}$, respectively. The lock-mass of leucine enkephalin via the LockSpray interface at 40 fmol $\mathrm{mL}^{-1}$ under a flow rate of $10 \mu \mathrm{L} \min ^{-1}$ was employed to ensure the accuracy and repeatability of the instrument during UPLC/MS.

2.6.3 Data processing. MarkerLynx Application Manager (Waters Corp.) was utilized to preprocess all the original UPLC-MS before multivariate data analysis, which involved detecting the chromatographic peak, maximizing ions, locating the maximized ions, and transforming the ion data into a matrix. The software recorded every peak in mapping the expressed retention time $\left(R_{\mathrm{t}}\right)$ and $\mathrm{m} / \mathrm{z}$ data pair. The ion intensities of each peak detected were normalized to the sum of the peak intensities in each blood sample, and afterwards they were enlarged 10000 times. The obtained data matrix contained a table of $R_{\mathrm{t}}, \mathrm{m} / \mathrm{z}$ and normalized chromatographic peak area calculated with a single accurate mass. The EZinfo 2.0 software (Waters Corporation, Manchester, UK) 
was used for the multivariate analysis to probe the similarities and differences in the lipids in the different groups. Principal component analysis (PCA), as an exploratory unsupervised pattern recognition method, can narrow the range of latent variables, which presents a grouping trend in the score plot when lipid compounds exhibit high similarity and vice versa. Further, to reduce the counterfeit separation of categories and focus on the diversity among the groups, orthogonal partial least-squares discriminant analysis (OPLS-DA), as an exploratory supervised pattern recognition method, was used after verifying the model by permutation test parameters and calculating the misclassification rate. The parameters contain $\mathrm{R} 2 \mathrm{X}, \mathrm{R} 2 \mathrm{Y}$, and Q2 for evaluating the goodness of suit and the predictive ability, where R2 Y and Q2 values close to 1.0 indicate that it is an excellent model. The S-plot and VIP-plot (Variable Importance in the Projection) were generated by OPLS analysis based on their contribution value to the alteration and connection ratio of CHD in the lipid metabolic profiling. Ions with a higher VIP value show a positive correlation to the contribution ratio between the control group and the CHD model group. The potential ions closely related with CHD were screened by limiting the VIP value to greater than 1 and $P$-value less than 0.05 in the Student's $t$-test, and then the chemical structure was identified by $R_{\mathrm{t}}$, exact molecular mass, and the Mass Fragment software using the MS/MS data. The selected lipid potential biomarkers were confirmed by the searching the Chemspider (http:/www.chemspider.com/), HMDB (http:// www.hmdb.org/), KEGG (http://www.genome.jp/kegg/), METLIN (http://metlin.scripps.edu/), and Lipid Maps (http://dev.lipidmaps.org) online databases. The relative signal intensities of selected lipids in the control, CHD model and Sal B treatment groups were counted to determine the turbulence of CHD at the lipidomics level and the therapeutic activity of Sal B on CHD animals. The pathway analysis and internal relationship between the lipid biomarkers were resolved by MetaboAnalyst 4.0 (http://www.metaboanalyst.ca/) and KEGG pathway (http://www.genome.jp/kegg/) to explore the concentration changes, interaction, metabolic pathways and networks. All the quantitative data were expressed as mean \pm SD after statistical analysis using SPSS Statistics 19.0 (SPSS, Chicago, IL, USA). A $p$ value less than 0.05 is significant between two groups, and a $p$ value less than 0.01 is extremely meaningful between two groups.

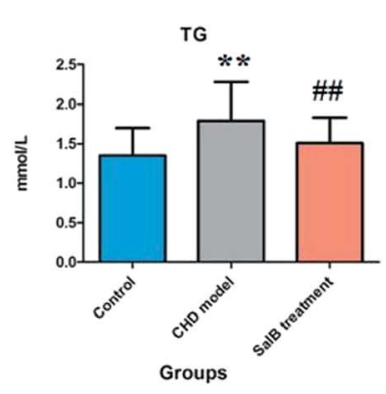

Apo (a1)

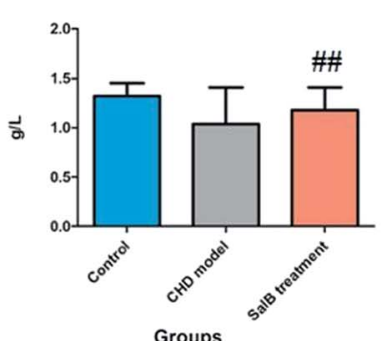

CK-MB

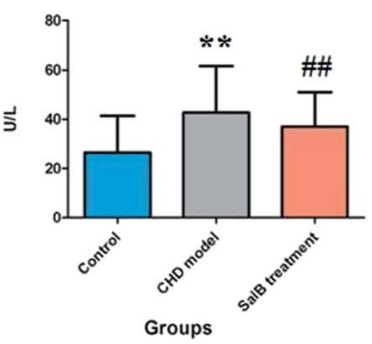

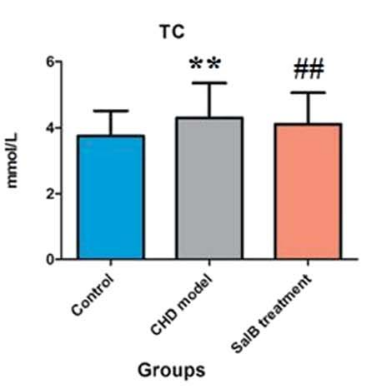

Apo (b)

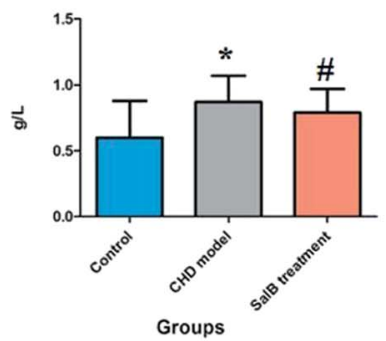

MYO

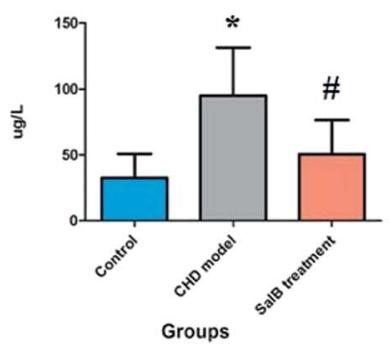

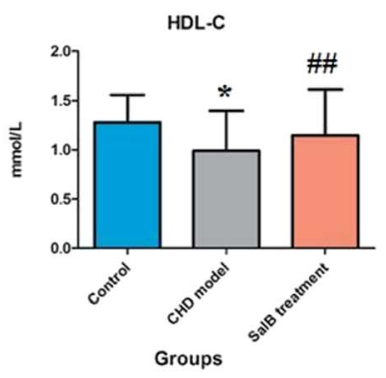

LDH

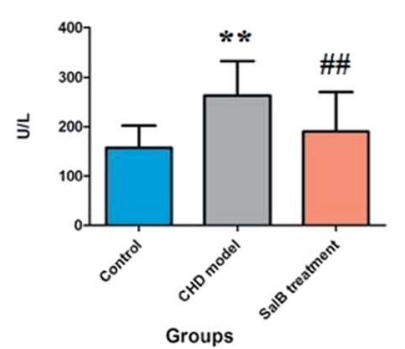

CTn1

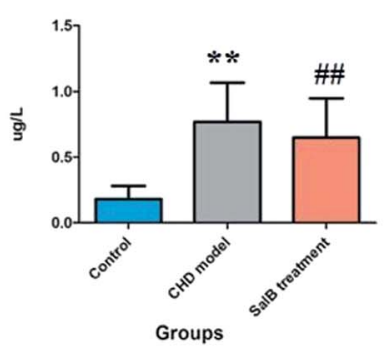

LDL-C

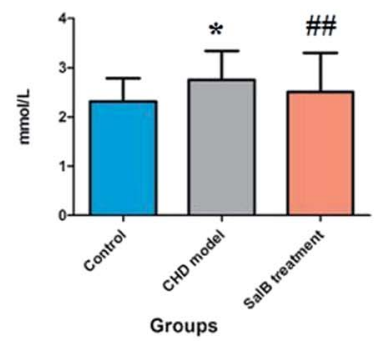

CK

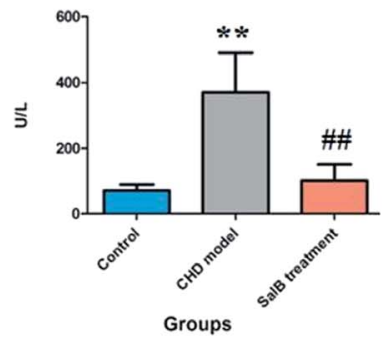

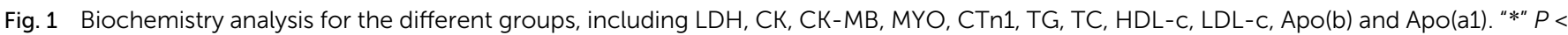
0.05; "**" $P<0.01$; "\#" $P<0.05$; "\#\#" $P<0.01$. 


\section{Results}

\subsection{The effect of Sal B on biochemical indexes}

The ELISA results for the biochemical indicators in the serum are shown in Fig. 1. Compared with the control group, the rats in the CHD model group treated by consecutive high-fat diet feeding with vitamin D3 injection showed an abnormal content of myocardial enzymes, troponin, blood lipids, and apolipoprotein, which indicated that the animals suffer from heart damage. In the CHD model, the level of LDH, CK, CK-MB, MYO, CTn1,TG, TC, LDL-c, and Apo(b) was higher than that in the healthy rats, and the level of HDL-c and Apo(a1) was lower than that in the healthy rats. Compared with the model group, the degree of myocardial injury was significantly relieved in the Sal B treatment group, its serum LDH, CK, CK-MB, CTn1, TG, TC, and LDL-c level was notably down-regulated with a $p$ value of less than 0.01, and the serum MYO and Apo(b) level was reduced more than that in the model group with a $p$ value of less than 0.05. Meanwhile, the level of HDL-c and Apo(a1) in the blood sample was significantly elevated compared to that in the model group with a $p$ value of less than 0.01 .

\subsection{Metabolic profiling analysis}

Lipidomics analysis of the blood sample gave a well-resolved peak shape, intensity, and separation in the total ion chromatograms (TICs) under the ESI+ and ESI- full-scan analysis modes. The trajectory analysis of the unsupervised PCA score plots in both modes is presented in Fig. 2, showing obvious visual separations among the control, CHD model and Sal B treatment groups. It indicates that the animal model of $\mathrm{CHD}$ caused by consecutive high-fat diet feeding with vitamin D3 injection was successfully established, and the clustering in the CHD model group exhibited the maximum separation trend compared to the control group, implying significant lipid metabolic alteration and pathobiological changes. In contrast, the Sal B treatment group was situated between the control and model group close to the control group, implying that Sal B can regulate the aberrant metabolism of lipids. In the preliminary experiment, the PCA result showed that $47.8 \%$ and $46.5 \%$ of the total variance can be explained by the first two principal components, which suggests that the analytical method has favorable repeatability and stability compared to the QC samples. The 3D OPLS-DA score plots acquired from the UPLC/ MS data under the positive and negative ion modes in Fig. 2 are

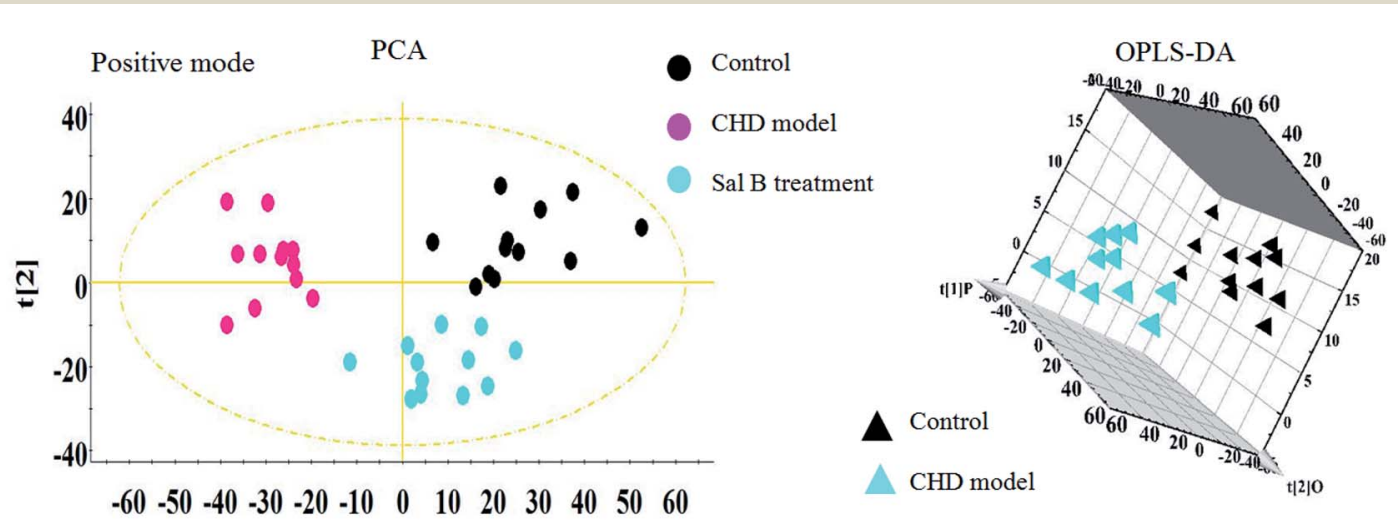

$\mathrm{t}[1]$
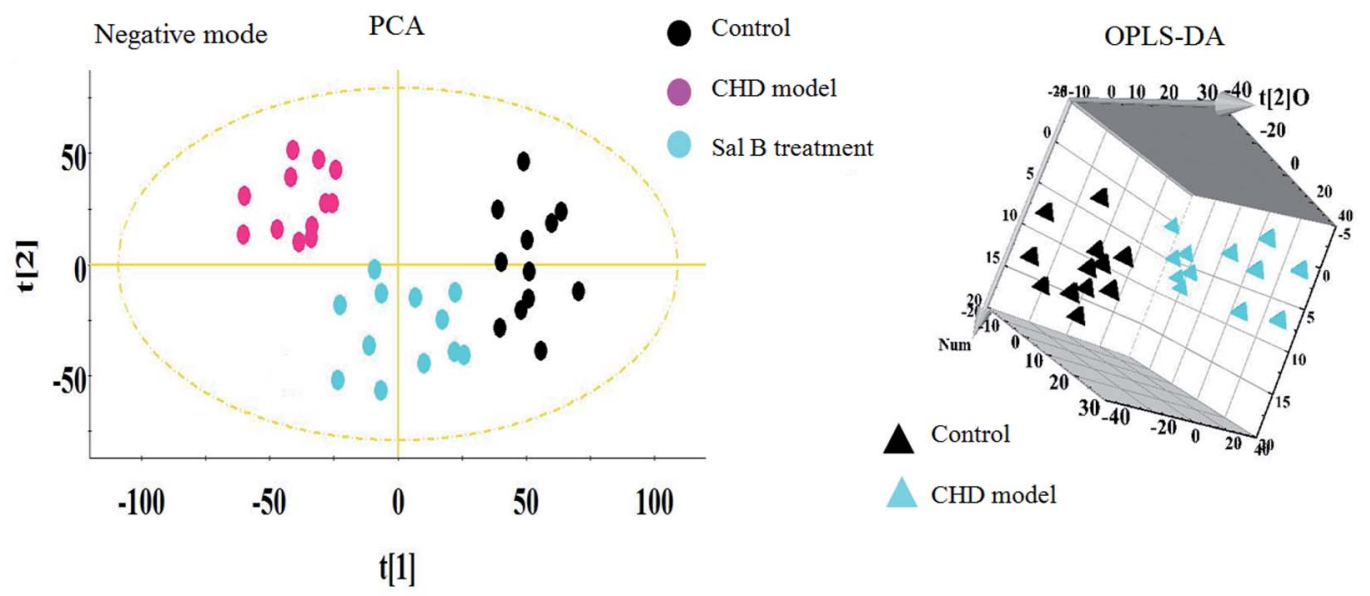

Fig. 2 Multivariate analysis from lipid metabolites in the CHD model before and after Sal B treatment. PCA score plot of lipid metabolites changes for clustering rats in the control, CHD model and Sal B group in positive ion mode and negative ion mode. 3D OPLS-DA score plot of lipid metabolites changes for clustering rats among the control, and CHD model group in positive ion mode and negative ion mode. 

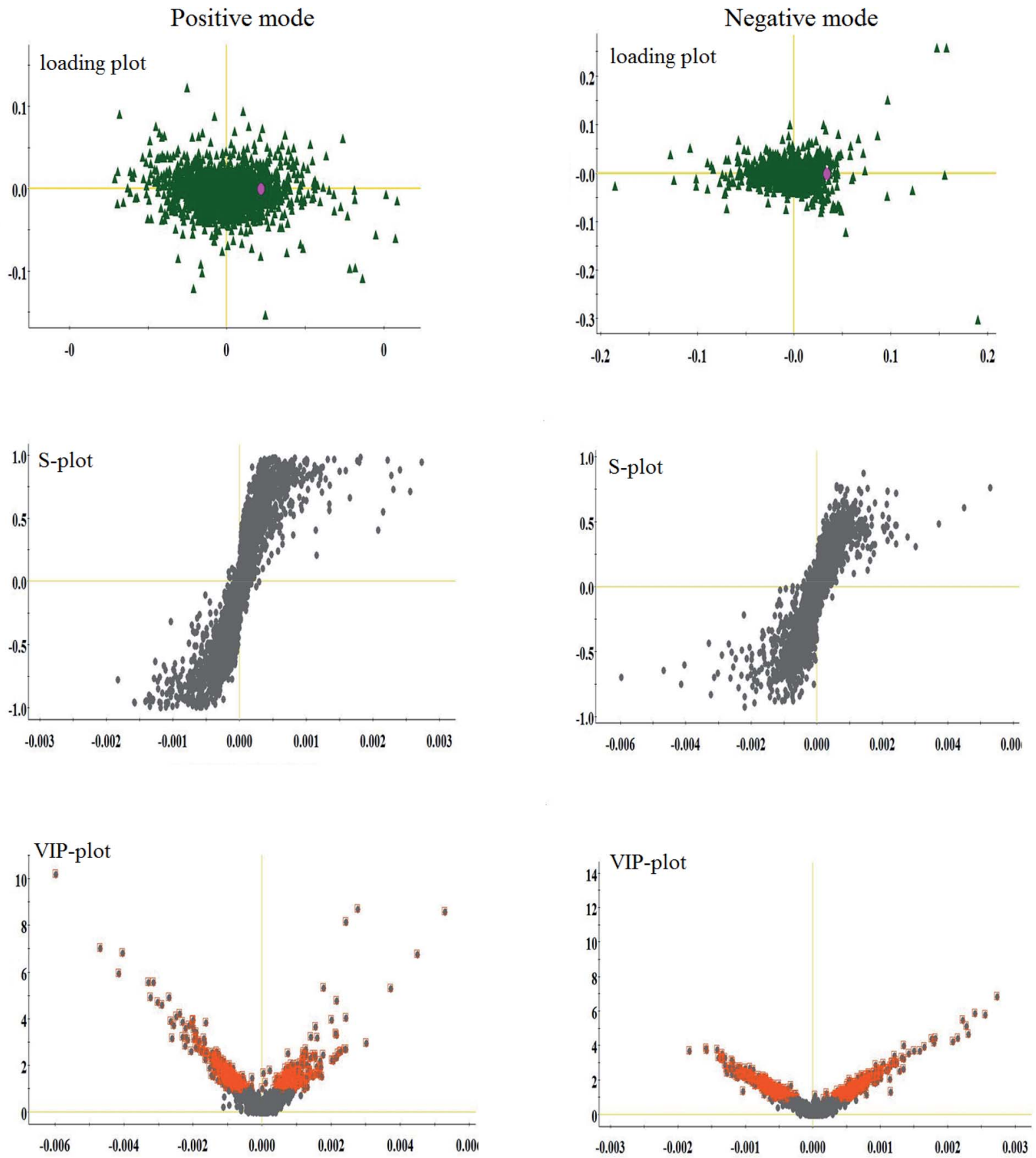

Fig. 3 Multivariate analysis from lipid metabolites in the CHD model before and after Sal B treatment. Loading plot, S-plot, and VIP-plot of OPLSDA model of serum metabolites for clustering the control and model groups in both ion modes.

obviously separated, which reveal the differences in the lipids between the control and CHD model groups. The R2 Y and Q2 parameters obtained by cross-validation in the OPLS-DA analysis were 0.915 and 0.705 in the positive mode and 0.923 and 0.737 in the negative mode, respectively. These results imply that the statistical models exhibit suitable goodness-of-fit and goodness-of-prediction for the data, respectively. In the corresponding loading plots shown in Fig. 3, the ions of the lipids that are furthest away from the origin are considered the differentiated lipids of the CHD rats.

\subsection{Identification of differential metabolites}

With the aim of determining the difference variables with great contributions for data classification between the healthy and CHD model rats, the S-plot and VIP score plot were obtained from the OPLS-DA analysis, as shown in Fig. 3, where each point in both plots represents an original variable. Metabolite ions with a threshold of $>1$ were selected by VIP in the OPLS-DA model and together with a $p$ value of less than 0.05 in the $t$ test, a total of twenty-six lipid metabolites involved in 


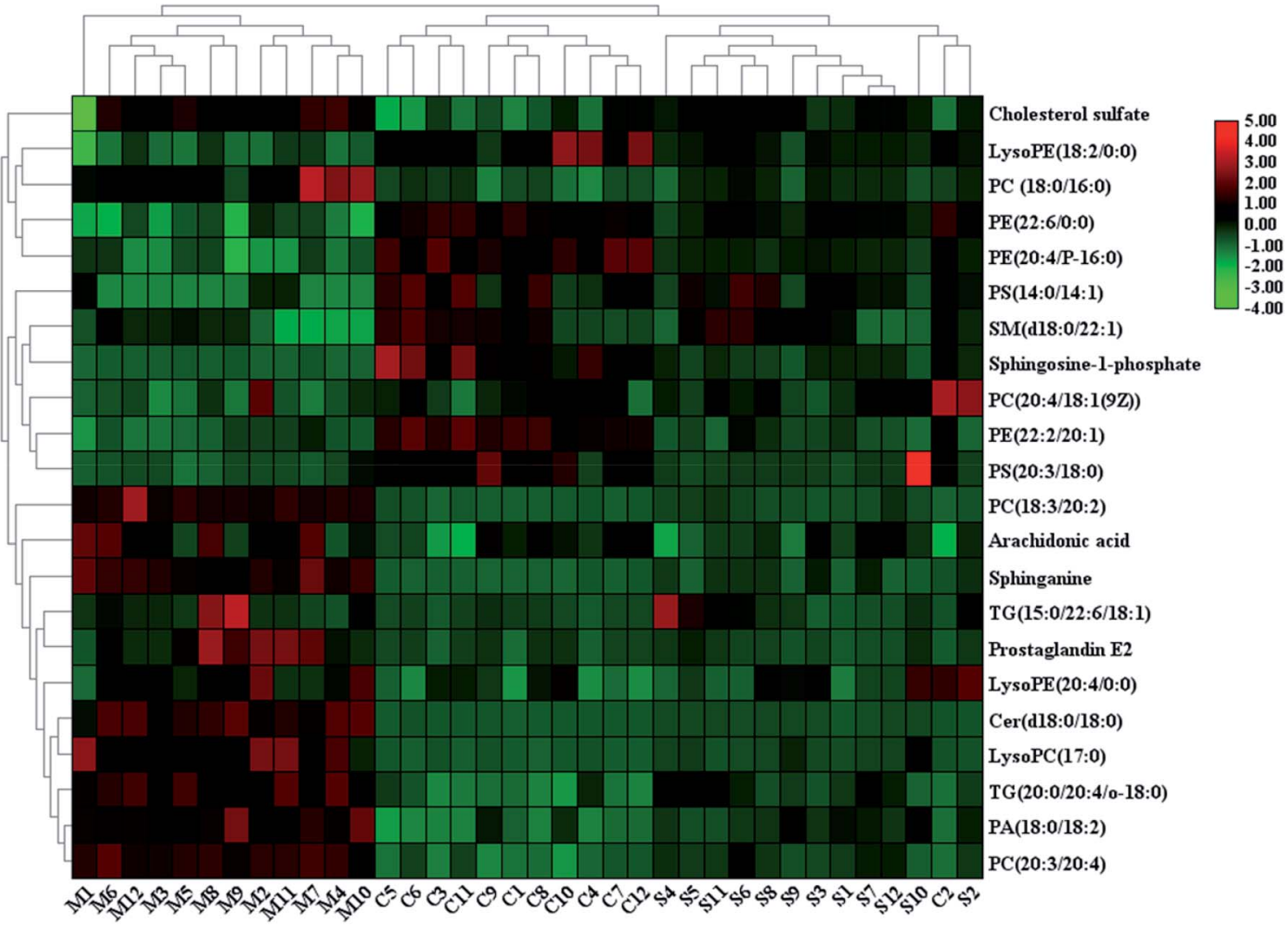

Fig. 4 Heatmap visualisation of the remarkable changes of the twenty-two potential lipid biomarkers in all the experimental animals among the control, CHD model and Sal B treatment groups. Red symbol indicates an increasing trend in serum level, green symbol indicates a decreasing trend in serum level, and black symbol indicates a trend in between.

glycerophospholipids, triacylglycerols, sphingolipids, sterol lipids and others were identified and detected in all three groups of rats, as shown in Table $S 1, \dagger$ which contains metabolite information such as ion mode, $R_{\mathrm{t}}(\mathrm{min})$, error, compound name and formula. After Sal B treatment, twenty-two significantly altered metabolites were observed in comparison with the CHD group, including TG(20:0/20:4/o-18:0), PC(20:4/ 18:1(9Z)), PC(18:3/20:2), PA(18:0/18:2), LysoPE(18:2/0:0), $\operatorname{SM}(\mathrm{d} 18: 0 / 22: 1), \operatorname{PE}(22: 6 / 0: 0)$, LysoPE(20:4/0:0), sphinganine, Cer(d18:0/18:0), PS(14:0/14:1), PC(18:0/16:0), LysoPC(17:0), $\operatorname{PE}(22: 2 / 20: 1), \quad \operatorname{PC}(20: 3 / 20: 4), \quad \operatorname{PE}(20: 4 / \mathrm{P}-16: 0), \quad \operatorname{PS}(20: 3 / 18: 0)$, cholesterol sulfate, TG(15:0/22:6/18:1), prostaglandin $\mathrm{E} 2$, arachidonic acid and sphingosine-1-phosphate. Nine metabolites including PC(20:4/18:1(9Z)), PS(14:0/14:1), PE(22:2/20:1), $\operatorname{PS}(20: 3 / 18: 0)$, sphingosine-1-phosphate, LysoPE (18:2/0:0), $\operatorname{SM}(\mathrm{d} 18: 0 / 22: 1), \quad \operatorname{PE}(22: 6 / 0: 0)$, and $\operatorname{PE}(20: 4 / \mathrm{P}-16: 0)$ showed a marked decrease and thirteen metabolites including TG(20:0/ 20:4/o-18:0), PC(18:3/20:2), PA(18:0/18:2), LysoPE(20:4/0:0), Cer(d18:0/18:0), LysoPC(17:0), PC(20:3/20:4), TG(15:0/22:6/ 18:1), prostaglandin E2, arachidonic acid, sphinganine, PC(18:0/16:0) and cholesterol sulfate showed a marked increase in the visualized heat-map (Fig. 4). According to Fig. 5, using UPLC-MS, the relative signal intensities of the identified lipids biomarkers indicate the relative changes in their concentration among the control, CHD model and Sal B treatment group.

\subsection{Metabolic pathways and network construction}

To determine and analyze the relationship among the selected lipids metabolites and the possible metabolic pathway for Sal B protecting against CHD, MetPA (Metabolomics Pathway Analysis) was established to disclose the most vital pathways due to the administration of Sal B. As shown in Fig. 6A, six metabolic pathways in the blood including glycerophospholipid metabolism, sphingolipid metabolism, arachidonic acid metabolism, glycosylphosphatidylinositol (GPI)-anchor biosynthesis, glycerolipid metabolism and phosphatidylinositol signaling were identified in the process of Sal B protecting against CHD after the Pareto method was employed to standardize the data. The metabolic pathway influence values were $0.3562,0.1785,0.3329$, $0.0040,0.0125$, and 0.0125 , respectively. According to the KEGG global metabolic network in Fig. 6B, which maps the metabolites in the model animals with CHD after Sal B administration and enzymes/KOs (KEGG Orthologs), the results were compared with common metabolomics and metagenomics studies mostly associated with glycerophospholipid metabolism, sphingolipid metabolism, arachidonic acid metabolism, glycosylphosphatidylinositol(GPI)-anchor biosynthesis, linoleic acid metabolism, alpha-linolenic acid metabolism, and glycerolipid metabolism. Due to the metabolic pathway influence value of more than 1 during the process of Sal B protecting against CHD, glycerophospholipid metabolism, sphingolipid metabolism 

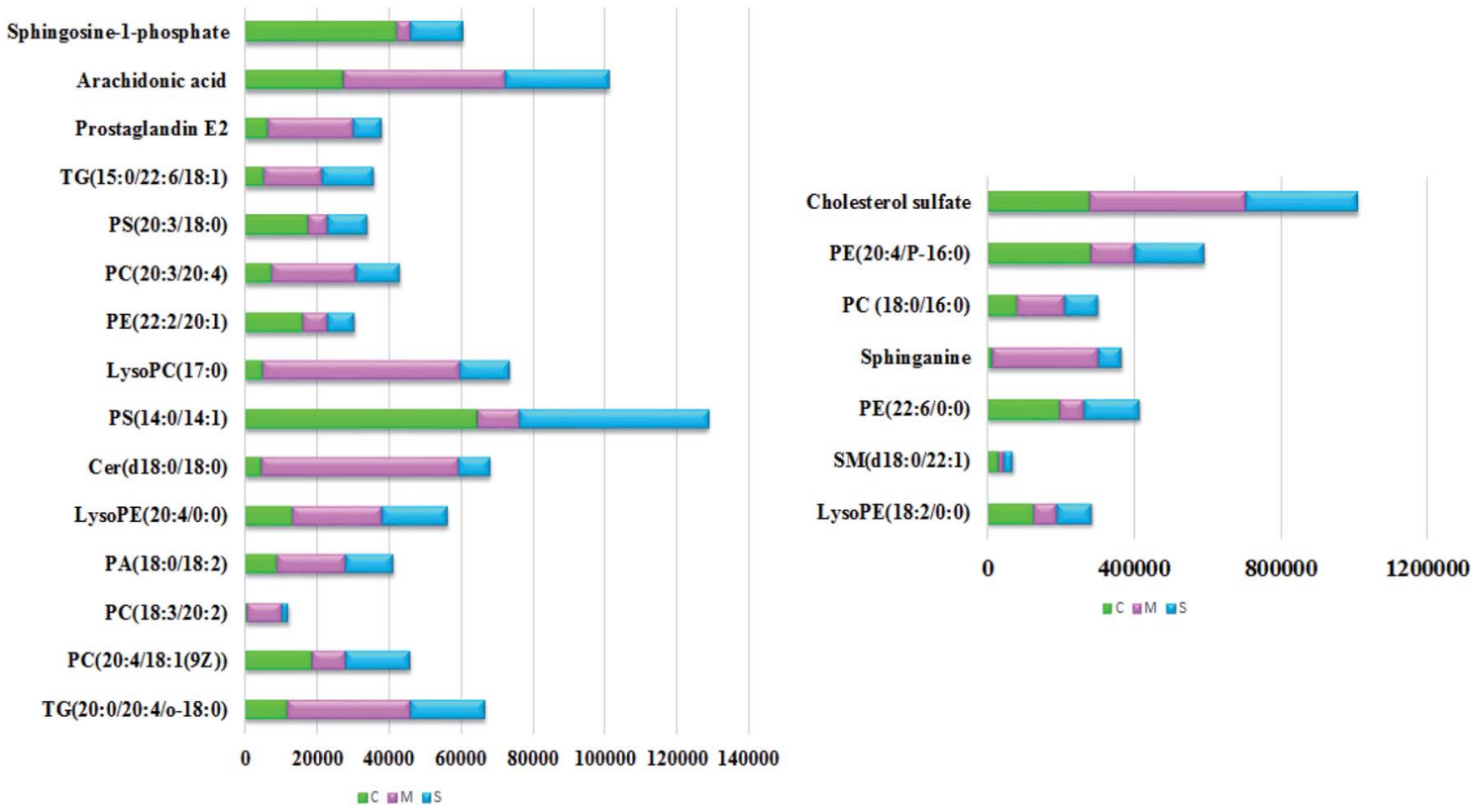

Fig. 5 Relative signal intensities of the lipid metabolites in the serum samples from the control, CHD model and Sal B treatment groups identified by UPLC-MS.

and arachidonic acid metabolism were considered as the key target pathways, and their KEGG network diagrams are shown in Fig. S1. $\dagger$ The result of enrichment and network analysis in Fig. S2 $\uparrow$ shows that the enzyme activity of carbonyl reductase, prostaglandin F2alpha exchange, ethanolamine phosphate demand, palmitate ER export, sphingosine-1-phosphate lyase, sphingosine-1-phosphate transport, fatty-acyl-CoA elongation, fatty acyl-CoA desaturase, sphingomyelin synthase, sphingosine kinase 2, intracellular transport, prostaglandin D2 exchange and others affects the pharmacodynamic effects of Sal B on $\mathrm{CHD}$, as predicted by the genome-scale network model of human metabolism. The relationship between the lipids and genes was exhibited, which highlighted arachidonate (57 genes), phosphatidylcholine (42 genes), phosphatidylethanolamine (34 genes), phosphatidate (34 genes), 1-acyl-sn-glycero-3phosphocholine (33 genes), sphinganine (23genes), sphingomyelin (13 genes), and sphingosine 1-phosphate (11 genes), providing the basic data for the precision medicine research of CHD. Subsequently, the single nucleotide polymorphism (SNP) loci of these lipid metabolites were detected based on their associations shown in Fig. S2.†

\section{Discussion}

Myocardial enzymes such as LDH, CK, and CK-MB are collective names of the various enzymes present in the myocardium, which are released to the serum in varying degrees after myocardial injury. When myocardial cells are necrotic and ruptured, LDH, CK, and CK-MB are released into the blood, leading to an increase in various indicators, which are used to measure the degree of damage to myocardial cells, judge the prognosis of patients, guide clinical medication, and observe the efficacy of treatment. ${ }^{27}$ CTn1 is a specific marker of myocardial necrosis with high specificity and sensitivity. An elevated level of CTn1 indicates the occurrence of cardiomyocyte necrosis. As a recognized good myocardial injury marker in the early stage of injury, MYO in the striated muscle of the heart and bones is quickly released into the blood when muscle cells are damaged, with an abnormal increase within 1 to $3 \mathrm{~h}$. Although myocardial specificity is not high, it is quickly released from the necrotic myocardium with high sensitivity in CHD. ${ }^{28,29}$ HDL-c, which is mainly synthesized in the liver, is a group of lipoproteins with the highest particle density in serum, reflecting high-density lipoprotein levels. HDL-c is inversely related to the incidence and severity of cardiovascular disease. LDL-c is converted from very low-density lipoprotein in the plasma, and is mainly synthesized in blood vessels. It is the main vehicle for transporting cholesterol to extrahepatic tissues, which is mainly used for the evaluation of lipid metabolism disorders and the risk prediction of $\mathrm{CHD}^{30,31}$ Apo(b), which is applied for cell recognition and uptake of LDL, exists on the surface of low-density lipoprotein. When the level of Apo(b) increases, it can increase the incidence of CHD even if the LDL level is normal. As the main structural protein of highdensity lipoprotein cholesterol (HDL-CHOL) accounting for $60 \%$ to $70 \%$, Apo(a1) is mainly synthesized by the liver and can also be synthesized in the small intestine, which can prevent CHD and atherosclerosis. Blood lipids are usually divided into two groups, triglycerides and cholesterol. High levels of triglycerides and total serum cholesterol increase the risk of heart disease..$^{32,33}$ The results of the biochemical analysis showed that compared with the healthy rats, the levels of LDH, CK, CK-MB, CTn1, TG, and TC in the blood of the model group were significantly different from that of the control group $(P<$ $0.01)$. Although there was no statistically significant difference in Apo(a1), the levels of MYO, HDL-c, LDL-c, and Apo(b) were all 
A
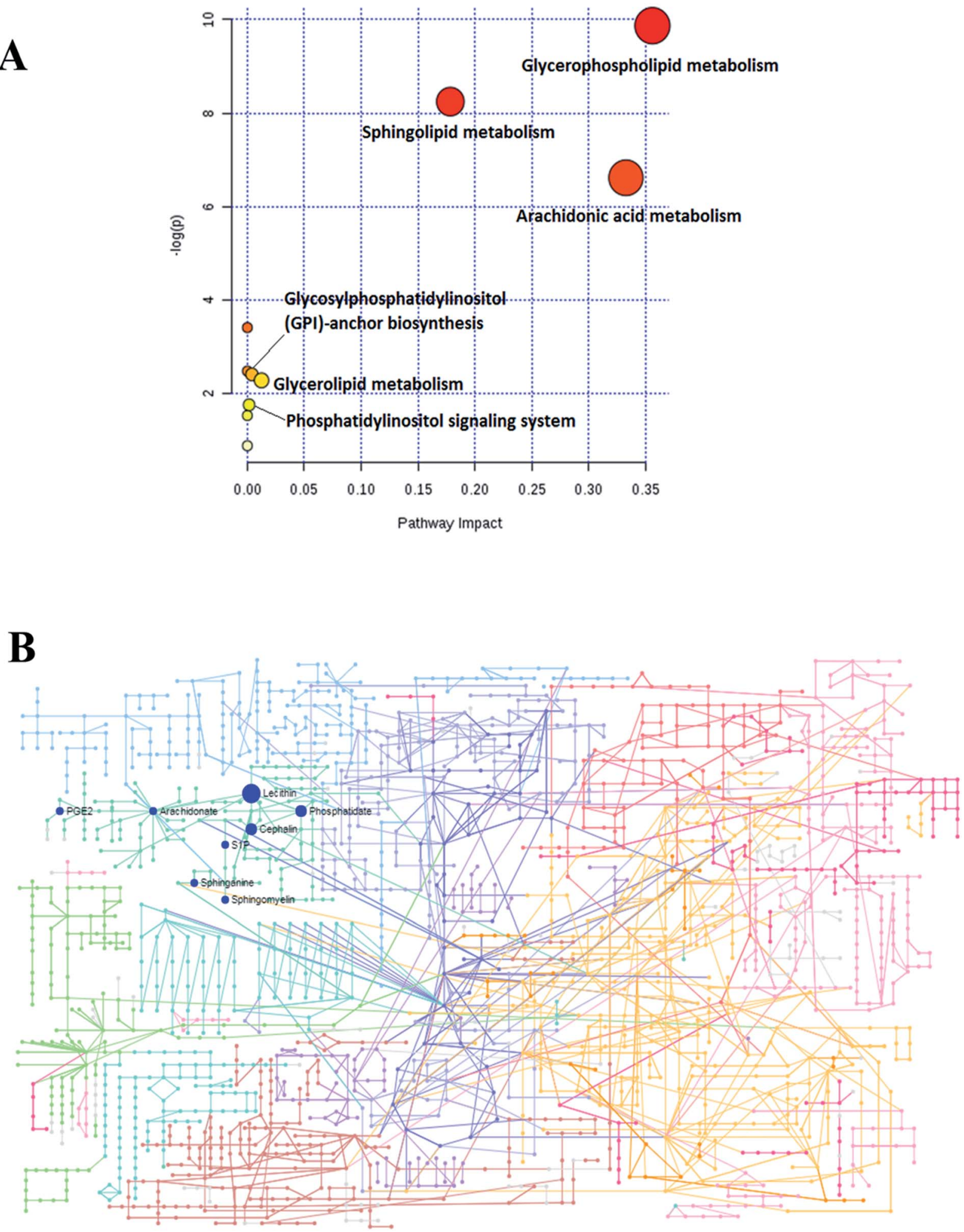

Fig. 6 (A) Changes in the metabolomic pathways observed in the serum sample after Sal B treatment. (B) KEGG global metabolic network associated with Sal B protective activity in CHD model.

different from that in the control group $(P<0.05)$. Thus, it can be concluded that the CHD rats have serious lipid metabolism abnormalities compared with the healthy rats, which is consistent with the myocardial histopathological results. Disturbance of lipid metabolism can be considered as one of the vital characteristics of CHD. After Sal B treatment, the serum 
LDH, CK, CK-MB, CTn1, TG, TC, and LDL-c content $(p<0.01)$ and MYO and Apo(b) $(p<0.05)$ were notably down-regulated, and the level of HDL-c and Apo(a1) $(p<0.01)$ was significantly elevated compared to that in the model group, indicating that Sal B has a therapeutic effect on CHD.

At the molecular level, we employed UPLC/MS to perform qualitative and quantitative analysis of the lipids in the blood between the healthy rats and CHD rats, including fatty acyls, glycerophospholipids, triacylglycerols, sphingolipids, sterol lipids, and others. Glycerophospholipid, which contains at least one fatty acyl side chain connected by acyl, alkyl, or alkenyl groups, is the most abundant lipid and the main component of bile and membrane surfactants besides biofilms, which are classified into phosphatidic acid (PA), phosphatidylglycerol (PG), phosphatidylinositol (PI), and phosphatidylcholine (PC), phosphatidylethanolamine (PE), and phosphatidylserine (PS), as well as lyso-PL after the hydrolysis of a fatty acid side chain according to the different polar heads. The metabolism of different phospholipids and their mutual transformation constitute a complex network, which is regulated by a variety of metabolic enzymes. As a synthetic precursor of other lipids, phosphatidic acid (PA) is a common phospholipid, which acts as a signal lipid molecule based on the curvature of the membrane due to its physical properties. It promotes atherosclerosis and thrombosis from many aspects with clinical significance. An elevated PA content is ascribed to platelet activation and can further lead to the waterfall-like activation of platelets, which is the basis of PA promoting thrombosis. Lowdensity lipoprotein cholesterol (LDL-C) produces a large amount of LPA during mild oxidation, stimulating the proliferation of vascular endothelial cells and increasing the interval of endothelial cells. Meanwhile, PA induces the migration of vascular smooth muscle cells from the middle layer to the intima, which promotes the development of atherosclerosis, leading to an important process of narrowing the lumen. In addition, PA also promotes the release of a large amount of endothelin, causing vasospasm. The process of atherosclerosis caused by an increased PA level is very important. It promotes the occurrence and development of plaques, which indicates that the body is in a hypercoagulable state, increasing the risk of thrombosis, and then may cause narrowing or blockage of the blood vessel cavity. ${ }^{34-36}$ PC and lysophosphatidylcholines (LPC), which belong to the glycerophosphate group, are mostly involved in glycerophospholipid metabolism. They can mutually transform into each other. Some studies have reported that lipid metabolism is regulated by peroxisome proliferator activated receptor $\gamma$ (PPAR $\gamma$ ), and the accumulation of lipids elevates their oxidation, resulting in the activation of PPAR $\gamma$. PPAR $\gamma$-plays a role in regulating fat metabolism, inflammation, immunity, and cell differentiation. In the blood, an increased content of LPC and PC suggests that an oxidative stress response was evoked. ${ }^{37}$ Phosphatidylserine (PS), also known as composite neural acid, is extracted from the residue of natural soybean oil. It is the active substance of the cell membrane, especially in brain cells. The biological function of PS is mainly to improve nerve cell action, regulate the transmission of neural pulses, and enhance brain memory. Due to its strong lipophilicity, it can quickly enter the brain through the bloodbrain barrier after absorption for soothing vascular smooth muscle cells and increasing the blood supply in the brain. ${ }^{38}$ The basic components of cerebral phospholipids (PE) are diglyceride, phosphoric acid, and choline (ethanolamine), which can be decarboxylated by serine in the body to generate choline, which activates human nerve cells and improves brain function. It heightens the speed and accuracy of information transmission between various nerve cells, and accelerates the further establishment and enrichment of information channels beyond that participating in the coagulation process. ${ }^{39}$ Glycosylphosphatidyl inositol (GPI), a protein-monosaccharide-fatty acid compound, is involved in proteins binding to cell membranes. GPI-anchored proteins such as CD24 and CD87 can adjust the adhesion and migration of leukocytes, while the invasion and adhesion of leukocytes to endothelial cells is one of the key early events from atherosclerosis to CHD, which is closely associated with $\operatorname{PE}(22: 2 / 20: 1)$ in the KEGG pathway result. $^{40}$ In this study, the concentration of seven glycerophosphatides obviously increased, including $\operatorname{PC}(18: 3 / 20: 2)$, PC(20:3/20:4), PC(18:0/16:0), PA(18:0/18:2), LysoPE(20:4/0:0), LysoPC(20:2) and LysoPC(17:0), and the concentration of eight glycerophosphatides obviously declined including $\mathrm{PE}(22: 6 / 0: 0)$, PE(20:4/P-16:0), PE(22:2/20:1), LysoPE(18:2/0:0), PS(14:0/14:1), $\operatorname{PS}(18: 0 / 18: 3), \operatorname{PS}(20: 3 / 18: 0)$ and $\operatorname{PC}(20: 4 / 18: 1)$ in the CHD model rats. Under normal physiological conditions, the amount of these lipids is not significant, but under inflammation, they can aggregate and produce obvious pathological features in glycerophospholipid. After Sal B treatment, the above biomarkers were relieved to varying degrees and tended to normal levels by adjusting glycerophospholipid metabolism, glycerolipid metabolism and glycosylphosphatidylinositol (GPI)-anchor biosynthesis except LysoPC(20:2) and PS(18:0/ 18:3).

Sphingomyelin (SM) is present in all major lipoproteins and is the second most abundant phospholipid in the body, which is composed of sphingosine, fatty acid, and choline phosphate. Its fatty acid chain forms a hydrophobic tail, and its hydrophilic head is composed of choline and phosphoric acid. It is a major component widely present in mammalian cell membranes, mainly distributed in the outer layer of cell membranes, nerve myelin and blood lipoproteins. Research has shown that the synthesis and transport deregulation of SM are closely linked with various types of metabolic disorders. ${ }^{41}$ Ceramide (Cers) are mainly synthesized via de novo synthesis and also produced by the degradation of sphingomyelin or cerebrosides. The level of Cer and sphingosine increases after ischemia reperfusion of the heart, in the infarction zone and in the blood, and also in hypertension. Sphingosine-1-phosphate (S-1-P) exhibits evident cardioprotective properties. In the process of $\mathrm{CHD}$, the upregulated content of Cer and sphingosine and down-regulated levels of S-1-P are developed as a key factor in the course of coronary sclerosis. ${ }^{\mathbf{4 2 , 4 3}}$ In this study, we obtained a similar result, where the level of $\operatorname{Cer}(\mathrm{d} 18: 0 / 18: 0)$ and sphinganine increased and the level of SM(d18:0/22:1) and S-1-P decreased. Sal B can effectively inhibit abnormal changes by regulating the metabolism of sphingolipids. Fatty acids are divided into 
saturated fatty acids and unsaturated fatty acids, and unsaturated fatty acids are divided into monounsaturated fatty acids and polyunsaturated fatty acids containing $\omega-3$ series, $\omega-6$ series, $\omega-7$ series, $\omega-9$ series and others according to the different positions and functions of their double bonds. Studies have found that unsaturated fatty acids lower cholesterol and low-density lipoprotein, resist platelet aggregation, and have no effect on high-density lipoprotein. In contrast, saturated fatty acids have a strong effect of increasing the levels of cholesterol and low-density lipoprotein. It has been reported that unsaturated fatty acids are more beneficial for human health than long-chain fatty acids, especially for cardiovascular and cerebrovascular diseases, which can promote the transformation of excess cholesterol in the body into bile acids, inhibit cholesterol deposition in blood vessels, and prevent the occurrence of CHD and arteriosclerosis. ${ }^{44,45} \omega-3$ unsaturated fatty acids are precursors of arachidonic acid metabolites, and $\omega-6$ unsaturated fatty acids are metabolized to produce pro-inflammatory mediators, while $\omega-3$ unsaturated fatty acids produce anti-inflammatory mediators, resulting in protective effects for cardiovascular disease. Prostaglandins (PGs) are a type of cyclooxygenase (COX) metabolites of arachidonic acid produced by a series of enzymatic reactions. They play an important regulatory function in the body and participate in physiological and pathophysiological processes including fever, inflammatory response, pain perception, glycolipid metabolism, reproductive activities and cell growth and differentiation. $G$ protein-coupled receptors such as EP1, EP2, EP3 and EP4 mediate the biological function of PGE2, among which EP1 receptor may be related to the pain-causing effect of PGE2. In addition, a large number of studies have found that PGE2 also plays a key role in water and salt metabolism and blood pressure regulation. Thus, the elevated levels of arachidonic acid and PGE2 in the CHD model animals compared with the healthy rats in this study suggests that the inflammatory response may be closely linked with CHD, which may be due to the imbalance of fatty acid metabolism in the body inducing non-infectious inflammation and abnormal activation of platelets, leading to CHD and thrombosis. ${ }^{46,47}$

Different metabolic mechanisms not only generate different metabolism but also affect the metabolite levels, which can change the phenotypes of CHD symptoms and syndrome appearance. Lipid abnormal metabolism is a primary cause of CHD compared with other biological metabolism. Lipidomics is a strong and comprehensive set of tools, strategies, and methods in the precision medicine of CHD, which when coordinated with genomics and other omic methodologies, can probe specific functions of lifestyle environment, exposure, metabolism and genome on human health. In 2016, the White House came up with the Precision Medicine Initiative (PMI) in the USA to initiate a new era of individualized therapy with concerted efforts by patients and medical staff. This program is devoted to the treatment of diseases such as cancer and diabetes with the aim of accelerating accurate awareness and targeted treatment of diseases at the genomic level. ${ }^{48}$ Precision medicine, as a new medical treatment mode, is based on individual medicine and developed from genomics, proteomics, bioinformatics, and large data from science and other interdisciplinary applications. Thus, precision medicine for CHD provides ideas about CHD risk prediction, prevention, use of drugs, therapeutic efficacy and the results, besides seeking new targets for therapeutic intervention and drug discoveries with emphasis on the potential information of DNA sequence variants. ${ }^{49}$ Functional lipidomics as a promising strategy can used for discovering biomarkers, evaluating the efficacy of natural products and revealing their pathological mechanism. ${ }^{50-56}$ However, despite the promise and future possibilities of precision medicine, it faces many challenges, such as the highthroughput sequencing of precision medicine not represent the full precision medicine; a decrease in the number of patients who benefit from the same drug result in the increasing costs in drug development; the need for more new technology research and development personnel and materials to support the in-depth study of disease pathogenesis; and personal privacy and ethical challenges.

\section{Conclusion}

In this work, an untargeted lipidomics strategy based on UPLC/ MS was employed to investigate the serum metabolic changes in a rat model with CHD before and after treatment with a natural product. Upon Sal B intervention, lesion severity was evidently alleviated. Twenty-two latent lipids biomarkers related to Sal B pharmacodynamics on CHD were identified, which primarily involved TG(20:0/20:4/o-18:0), PC(20:4/18:1(9Z)), PC(18:3/20:2), $\operatorname{PA}(18: 0 / 18: 2), \operatorname{LysoPE}(18: 2 / 0: 0), \operatorname{SM}(\mathrm{d} 18: 0 / 22: 1), \operatorname{PE}(22: 6 / 0: 0)$, LysoPE(20:4/0:0), sphinganine, $\operatorname{Cer}(\mathrm{d} 18: 0 / 18: 0), \operatorname{PS}(14: 0 / 14: 1)$, $\operatorname{PC}(18: 0 / 16: 0), \quad$ LysoPC(17:0), $\quad \operatorname{PE}(22: 2 / 20: 1), \quad \operatorname{PC}(20: 3 / 20: 4)$, $\mathrm{PE}(20: 4 / \mathrm{P}-16: 0), \quad \mathrm{PS}(20: 3 / 18: 0)$, cholesterol sulfate, TG(15:0/ 22:6/18:1), prostaglandin E2, arachidonic acid and sphingosine-1-phosphate. Based on the evaluation of histological characteristics and biochemical indexes coupled with lipidomic profiling, it was proposed that CHD caused by high-fat diet feeding with vitamin D3 injection can be reversed to some extent by Sal B regulating glycerophospholipid metabolism, sphingolipid metabolism and arachidonic acid metabolism. Furthermore, the glycerophospholipid metabolism pathway may be a promising target for Sal B protection against CHD during the process of lipid metabolism disorder, which needs further research to be proven in the future.

\section{Conflicts of interest}

There are no conflicts to declare.

\section{Acknowledgements}

National Natural Science Foundation of China (81703710), Tianjin Research Program of Application Foundation and Advanced Technology (17JCYBJC41800), Tianjin Research Program of Application Foundation and Advanced Technology (19JCTPJC56900), Tianjin Municipal Bureau of Labor and Social Security (2018018). 


\section{References}

1 J. F. Polak, P. Ouyang, D. Vaidya, et al., Total brachial artery reactivity and first time incident coronary heart disease events in a longitudinal cohort study: the multi-ethnic study of atherosclerosis, PLoS One, 2019, 14(4), e0211726.

2 B. Cybulska and L. Kłosiewicz-Latoszek, Landmark studies in coronary heart disease epidemiology. The Framingham Heart Study after 70 years and the Seven Countries Study after 60 years, Kardiol. Pol., 2019, 77(2), 173-180.

3 S. Bodkhe, S. U. Jajoo, U. N. Jajoo, et al., Epidemiology of confirmed coronary heart disease among population older than 60 years in rural central India-a community-based cross-sectional study, Indian Heart J., 2019, 71(1), 39-44.

4 I. Dhar, G. F. T. Svingen, E. R. Pedersen, et al., Plasma cystathionine and risk of acute myocardial infarction among patients with coronary heart disease: results from two independent cohorts, Int. J. Cardiol., 2018, 266, 24-30.

5 S. C. Chang, M. Glymour, M. Cornelis, et al., Social Integration and Reduced Risk of Coronary Heart Disease in Women: The Role of Lifestyle Behaviors, Circ. Res., 2017, 120(12), 1927-1937.

6 G. D. Batty, M. Kivimäki and S. Bell, Comparison of risk factors for coronary heart disease morbidity versus mortality, Eur. J. Prev. Cardiol., 2019, 2047487319882512.

7 K. Kotseva, D. De Bacquer, C. Jennings, et al., Time Trends in Lifestyle, Risk Factor Control, and Use of Evidence-Based Medications in Patients With Coronary Heart Disease in Europe: Results From 3 EUROASPIRE Surveys, 1999-2013, Glob Heart, 2017, 12(4), 315-322.

8 Y. M. Wang, Q. Liu, W. H. Fu, et al., A rapid and efficient approach based on ultra-high liquid chromatography coupled with mass spectrometry for identification in vitro and in vivo constituents from shizao decoction, Phcog. Mag., 2020, 16(67), 148-155.

9 B. A. Kotsias, Curriculum vitae of Nobel laureate Youyou Tu, Rev. Med. Vet. (B. Aires), 2016, 76(2), 126-127.

10 M. A. Katary, R. Abdelsayed, A. Alhashim, et al., Salvianolic Acid B Slows the Progression of Breast Cancer Cell Growth via Enhancement of Apoptosis and Reduction of Oxidative Stress, Inflammation, and Angiogenesis, Int. J. Mol. Sci., 2019, 20(22), E5653.

11 Y. Li, L. Wang, Z. Dong, et al., Cardioprotection of salvianolic acid $\mathrm{B}$ and ginsenoside Rg1 combination on subacute myocardial infarction and the underlying mechanism, Phytomedicine, 2019, 57, 255-261.

12 Z. Qiao and Y. Xu, salvianolic acid b alleviating myocardium injury in ischemia reperfusion rats, Afr. J. Tradit., Complementary Altern. Med., 2016, 13(4), 157-161.

13 R. Zhou, H. Long, B. Zhang, et al., Salvianolic acid B, an antioxidant derived from Salvia militarize, protects mice against $\gamma$-radiation-induced damage through Nrf2/Bach1, Mol. Med. Rep., 2019, 19(2), 1309-1317.

14 Y. W. Lee, D. H. Kim, S. J. Jeon, et al., Neuroprotective effects of salvianolic acid $\mathrm{B}$ on an A $\beta 25-35$ peptide-induced mouse model of Alzheimer's disease, Eur. J. Pharmacol., 2013, 704(1-3), 70-77.

15 J. Wang, X. Xiong and B. Feng, Cardiovascular effects of salvianolic acid B, J. Evidence-Based Complementary Altern. Med., 2013, 2013, 247948.

16 C. Zhang, K. Wang, L. Yang, et al., The role of proinflammatory cytokines in lipid metabolism of metabolic diseases, Int. Rev. Immunol., 2019, 38(6), 249-266.

17 F. J. Brunner, C. Waldeyer, F. Ojeda, et al., Application of non-HDL cholesterol for population-based cardiovascular risk stratification: results from the multinational cardiovascular risk consortium, Lancet, 2019, (19), 32519.

18 A. Zhang, S. Qiu, H. Sun, T. Zhang, Y. Guan, Y. Han, G. Yan and $\mathrm{X}$. Wang, Scoparone affects lipid metabolism in primary hepatocytes using lipidomics, Sci. Rep., 2016, 6, 28031.

19 W. Li, H. Zhang, X. Zhou, et al., High-throughput liquid chromatography mass-spectrometry-driven lipidomics discover metabolic biomarkers and pathways as promising targets to reveal the therapeutic effects of the Shenqi pill, RSC Adv., 2020, 10(4), 2347-2358.

20 A. M. Campos, E. Maciel, A. S. Moreira, et al., Lipidomics of Mesenchymal Stromal Cells: Understanding the Adaptation of Phospholipid Profile in Response to Pro-Inflammatory Cytokines, J. Cell. Physiol., 2016, 231(5), 1024-1032.

21 S. Qiu, A. Zhang, Y. Guan, et al., Functional metabolomics using UPLC-Q/TOF-MS combined with ingenuity pathway analysis as a promising strategy for evaluating the efficacy and discovering amino acid metabolism as a potential therapeutic mechanism-related target for geniposide against alcoholic liver disease, RSC Adv., 2020, 10(5), 2677-2690.

22 P. Zabielski, H. R. Hady, M. Chacinska, et al., The effect of high fat diet and metformin treatment on liver lipids accumulation and their impact on insulin action, Sci. Rep., 2018, 8(1), 7249.

23 A. H. Zhang, Z. M. Ma, L. Kong, et al., High-throughput lipidomics analysis to discover lipid biomarkers and profiles as potential targets for evaluating efficacy of KaiXin-San against APP/PS1 transgenic mice based on UPLCQ/TOF-MS, Biomed. Chromatogr., 2020, 34(2), e4724.

24 M. Barchuk, A. Dutour, P. Ancel, et al., Untargeted Lipidomics Reveals a Specific Enrichment in Plasmalogens in Epicardial Adipose Tissue and a Specific Signature in Coronary Artery Disease, Arterioscler., Thromb., Vasc. Biol., 2020, ATVBAHA120313955.

25 G. V. Halade, A. Dorbane, K. A. Ingle, et al., Comprehensive targeted and non-targeted lipidomics analyses in failing and non-failing heart, Anal. Bioanal. Chem., 2018, 410(7), 19651976.

26 A. H. Zhang, Z. M. Ma, L. Kong, et al., High-throughput lipidomics analysis discovers lipid biomarkers and profiles as potential targets to evaluating efficacy of Kai-Xin-San against APP/PS1 transgenic mice based on UPLC-Q/TOFMS, Biomed. Chromatogr., 2019, e4724.

27 Z. T. Wei and J. X. Zhao, Clinical observation on effects of Fuzheng Jiedu Granule in treating coronary heart diseasepatients in long term contact with nickel, Zhongguo Zhongxiyi Jiehe Zazhi, 2007, 27(5), 418-420. 
28 H. Feng, Z. Wang, C. Wang, et al., Effect of Furostanol Saponins from Allium Macrostemon Bunge Bulbs on Platelet Aggregation Rate and PI3K/Akt Pathway in the Rat Model of Coronary Heart Disease, J. Evidence-Based Complementary Altern. Med., 2019, 2019, 9107847.

29 Z. Sun, W. Wu, J. Liu, et al., Influence of glucose-lowering rate on CKMB and myoglobin serum levels in type-2 diabetes patients with coronary heart disease, Hum. Immunol., 2014, 75(12), 1182-1187.

30 Q. Zhao, J. Li, J. Yang, et al., Association of total cholesterol and HDL-C levels and outcome in coronary heart disease patients with heart failure, Medicine, 2017, 96(9), e6094.

31 Q. Y. Xiang, F. Tian, Q. Z. Lin, et al., Comparison of remnant cholesterol levels estimated by calculated and measured LDL-C levels in Chinese patients with coronary heart disease, Clin. Chim. Acta, 2019, (19), 32056.

32 J. C. van Capelleveen, A. E. Bochem, S. M. Boekholdt, et al., Association of High-Density Lipoprotein-Cholesterol Versus Apolipoprotein A-I With Risk of Coronary Heart Disease: The European Prospective Investigation Into CancerNorfolk Prospective Population Study, the Atherosclerosis Risk in Communities Study, and the Women's Health Study, J. Am. Heart Assoc., 2017, 6(8), e006636.

33 M. Ala-Korpela, The culprit is the carrier, not the loads: cholesterol, triglycerides and apolipoprotein $\mathrm{B}$ in atherosclerosis and coronary heart disease, Int. J. Epidemiol., 2019, 48(5), 1389-1392.

34 M. A. Zhukovsky, A. Filograna, A. Luini, et al., Phosphatidic acid in membrane rearrangements, FEBS Lett., 2019, 593(17), 2428-2451.

35 P. S. Tappia, C. H. Yu, P. Di Nardo, et al., Depressed responsiveness of phospholipase $\mathrm{C}$ isoenzymes to phosphatidic acid in congestive heart failure, J. Mol. Cell. Cardiol., 2001, 33(3), 431-440.

36 V. T. Dang, A. Huang, L. H. Zhong, et al., Comprehensive Plasma Metabolomic Analyses of Atherosclerotic Progression Reveal Alterations in Glycerophospholipid and Sphingolipid Metabolism in Apolipoprotein E-deficient Mice, Sci. Rep., 2016, 6, 35037.

37 R. Angelini, G. Vortmeier, A. Corcelli, et al., A fast method for the determination of the PC/LPC ratio in intact serum by MALDI-TOF MS: an easy-to-follow lipid biomarker of inflammation, Chem. Phys. Lipids, 2014, 183, 169-175.

38 Y. Kou, L. Zou, R. Liu, et al., Intravascular cells and circulating microparticles induce procoagulant activity via phosphatidylserine exposure in heart failure, J. Thromb. Thrombolysis, 2019, 48(2), 187-194.

39 L. S. May-Zhang, Z. Chen, N. S. Dosoky, et al., Administration of N-Acyl-Phosphatidylethanolamine Expressing Bacteria to Low Density Lipoprotein Receptor-/-Mice Improves Indices of Cardiometabolic Disease, Sci. Rep., 2019, 9(1), 420.

40 B. Pode-Shakked, G. Heimer, T. Vilboux, et al., Cerebral and portal vein thrombosis, macrocephaly and atypical absence seizures in Glycosylphosphatidyl inositol deficiency due to a PIGM promoter mutation, Mol. Genet. Metab., 2019, 128(1-2), 151-161.
41 R. N. Lemaitre, P. N. Jensen, A. Hoofnagle, et al., Plasma Ceramides and Sphingomyelins in Relation to Heart Failure Risk, Circ.: Heart Failure, 2019, 12(7), e005708.

42 A. M. Fretts, P. N. Jensen, A. Hoofnagle, et al., Plasma Ceramide Species Are Associated with Diabetes Risk in Participants of the Strong HeartStudy, J. Nutr., 2019, nxz259.

43 A. Polzin, K. Piayda, P. Keul, et al., Plasma sphingosine-1phosphate concentrations are associated with systolic heart failure in patients with ischemic heart disease, $J$. Mol. Cell. Cardiol., 2017, 110, 35-37.

44 R. N. Lemaitre, B. McKnight, N. Sotoodehnia, et al., Circulating Very Long-Chain Saturated Fatty Acids and Heart Failure: The Cardiovascular Health Study, J. Am. Heart Assoc., 2018, 7(21), e010019.

$45 \mathrm{~J}$. Scholl, Coronary heart dieseas: Unsaturated fatty acids aren't a panacea, Dtsch. Med. Wochenschr., 2016, 141(14), 1001.

46 P. Harding and D. B. Murray, The contribution of prostaglandins versus prostacyclin in ventricular remodeling during heartfailure, Life Sci., 2011, 89(19-20), 671-676.

47 T. Przygodzki, M. Talar, P. Przygodzka, et al., Inhibition of cyclooxygenase-2 causes a decrease in coronary flow in diabetic mice. The possible role of PGE2 and dysfunctional vasodilation mediated by prostacyclin receptor, J. Physiol. Biochem., 2015, 71(3), 351-358.

48 S. Jaffe, Planning for US Precision Medicine Initiative underway, Lancet, 2015, 385(9986), 2448-2449.

49 S. H. Shah, D. Arnett, S. R. Houser, et al., Opportunities for the Cardiovascular Community in the Precision Medicine Initiative, Circulation, 2016, 133(2), 226-231.

50 T. Infante, L. Del Viscovo, M. L. De Rimini, et al., Network Medicine: A Clinical Approach for Precision Medicine and Personalized Therapy in Coronary Heart Disease, $J$. Atheroscler. Thromb., 2020, 27(4), 279-302.

51 H. L. Gao, A. H. Zhang, J. B. Yu, H. Sun, L. Kong, X. Q. Wang, G. L. Yan, L. Liu and X. J. Wang, High-throughput lipidomics characterize key lipid molecules as potential therapeutic targets of Kaixinsan protects against Alzheimer's disease in APP/PS1 transgenic mice, J. Chromatogr. B: Anal. Technol. Biomed. Life Sci., 2018, 1092, 286-295.

52 C. Liu, W. Zong, A. Zhang, et al., Lipidomic characterisation discovery for coronary heart disease diagnosis based on high-throughput ultra-performance liquid chromatography and mass spectrometry, RSC Adv., 2018, 8, 647-654.

53 Q. Liang, Y. Zhu, H. Liu, et al., High-throughput lipidomics enables discovery of the mode of action of huaxian capsule impacting the metabolism of sepsis, $R S C A d v$., 2017, 7(71), 44990-44996.

54 H. Zhang, A. Zhang, X. Zhou, et al., High-throughput lipidomics reveal mirabilite regulating lipid metabolism as anticancer therapeutics, RSC Adv. , 2018, 8(62), 35600-35610.

55 H. Sun, A. Zhang, G. Yan, et al., Metabolomic analysis of key regulatory metabolites in hepatitis $\mathrm{C}$ virus-infected tree shrews, Mol. Cell. Proteomics, 2013, 12(3), 710-719.

56 Q. Liang, C. Wang, B. Li, et al., Lipidomics Analysis Based on Liquid Chromatography Mass Spectrometry for Hepatocellular Carcinoma and Intrahepatic Cholangiocarcinoma, RSC Adv., 2015, 5, 63711-63718. 